\title{
On Nature of the Induced Anisotropy in the Chemically Deposited Co-P Films
}

\author{
Anatoly V. Chzhan ${ }^{1,2, *}$, Sergey A. Podorozhnyak ${ }^{1}$, Mikhail N. Volochaev ${ }^{3}$, and Gennady S. Patrin ${ }^{1,3}$ \\ ${ }^{1}$ Siberian Federal University, Krasnoyarsk, 660041 Russia \\ ${ }^{2}$ Krasnoyarsk State Agrarian University, Krasnoyarsk, 66049 Russia \\ ${ }^{3}$ Kirensky Institute of Physics, Federal Research Center KSC SB RAS, Krasnoyarsk, 660036 Russia
}

\begin{abstract}
The induced magnetic anisotropy in the Co-P films prepared by chemical deposition from liquid solutions in a magnetic field has been investigated. The pronounced difference between the anisotropy values and relaxation times points out the existence of different physical mechanisms responsible for the occurrence of anisotropy of the $\mathrm{Co}-\mathrm{P}$ films at low and high $\mathrm{pH}$ values. Possible mechanisms of the induced anisotropy in the Co-P films prepared at different $\mathrm{pH}$ values are discussed.
\end{abstract}

\section{Introduction}

The magnetic anisotropy is one of the fundamental properties of ferromagnets, since it strongly affects characteristics of these materials. In contrast to the crystallographic anisotropy, the induced anisotropy can be created artificially during fabrication of samples or upon subsequent temperature annealing in an applied magnetic field. The nature of induced anisotropy and its effect on the magnetic properties of samples are often arguable. This is caused by the existence of different physical mechanisms responsible for the occurrence of the induced anisotropy. Therefore, elucidation of the anisotropy origin needs thorough investigations.

The aim of this study was to determine the effect of acidity of working solutions on the value and dynamics of induced anisotropy in the Co-P films prepared by chemical deposition.

\section{Experimental}

Thin-film samples for investigations were formed by chemical reduction of $\mathrm{Co}$ and $\mathrm{P}$ from aqueous solutions [1]. The films were deposited onto glass substrates in a dc magnetic field of $\mathrm{H}=2.5 \mathrm{kOe}$, applied to the film's plane. The film thickness was $\sim 30 \mathrm{~nm}$. The solution acidity was changed by adding alkalies in different concentrations [2].

The film thickness and composition were determined by X-ray spectral analysis. The film microstructure was studied on a Hitachi HT-7700 transmission electron microscope (TEM) equipped with a Bruker X-Flash $6 \mathrm{~T} / 60$ energy-dispersive detector.

The constant of induced anisotropy $\mathrm{K}_{\mathrm{U}}$ is determined using a rotational anisometer in magnetic field $\mathrm{H}=10$ kOe. The anisotropy field $\mathrm{H}_{\mathrm{K}}$ is detected through analyzing the magnetic hysteresis loops at the frequency of $50 \mathrm{~Hz}$. To establish time evolution of the induced anisotropy, the films were additionally annealed in a dc magnetic field of $\mathrm{H}=3 \mathrm{kOe}$ in the direction perpendicular to the easy magnetization axis.

\section{Results}

Figure 1 shows the dependence of the induced anisotropy constant $\mathrm{K}_{\mathrm{U}}$ and coercivity $\mathrm{H}_{\mathrm{C}}$ on the solution acidity.

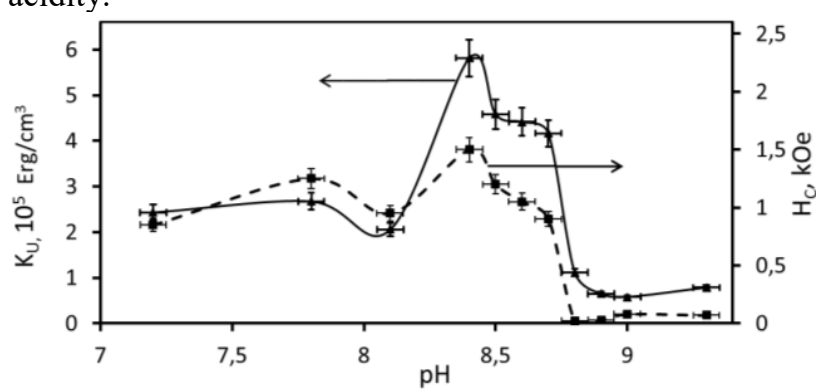

Fig. 1. Dependence of $\mathrm{K}_{U}$ and $\mathrm{H}_{\mathrm{C}}$ on the solution acidity.

In the low-pH region, we have $\mathrm{K}_{\mathrm{U}} \sim 2.5 \times 10^{5}$ $\mathrm{erg} / \mathrm{cm}^{3}$. As the $\mathrm{pH}$ value increases $(\mathrm{pH}>7,2)$, the anisotropy grows and attains about $6 \times 10^{5} \mathrm{erg} / \mathrm{cm}^{3}$ in the vicinity of $\mathrm{pH} \sim 8.5$. With a further increase in the acidity value, the $\mathrm{K}_{\mathrm{U}}$ value abruptly decreases up to $5 \times 10^{4} \mathrm{erg} / \mathrm{cm}^{3}$.

Simultaneously with $\mathrm{pH}$ growth up to $\sim 8.5, \mathrm{H}_{\mathrm{C}}$ increases from $1 \mathrm{kOe}$ to $1.5 \mathrm{kOe}$, and then $\mathrm{H}_{\mathrm{C}}$ abruptly drops stepwise up to several oersteds with further $\mathrm{pH}$ growth.

The saturation field $\mathrm{H}_{\mathrm{S}}$ in the films with uniaxial anisotropy being magnetized along the light direction practically coincides with the value of $\mathrm{H}_{\mathrm{C}}$, caused by the high degree of the hysteresis loop's rectangularity.

The observed variation in the induced anisotropy is consistent with the behavior of coercivity. As we 
demonstrated in [3], the high-coercivity region corresponds to the submicrocrystalline film structure, while the low-coercivity region corresponds to the nanocrystalline. The region of sharp coercivity variations corresponds to the transitions between these structures.

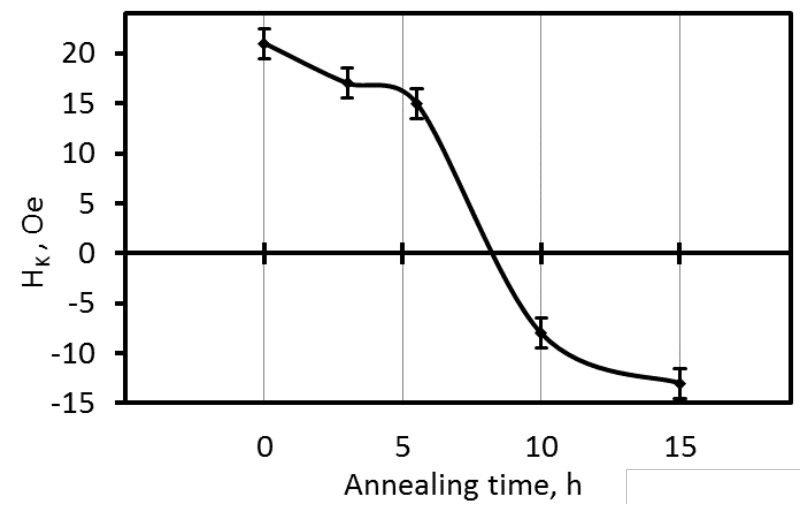

Fig. 2. Dependence of the anisotropy field in the Co-P films on the annealing time. The annealing temperature is $100^{\circ} \mathrm{C}$.

The difference in the nature of induced anisotropy in the films formed at the low and high $\mathrm{pH}$ values can be followed not only from the anisotropy values, but also from the dynamics of the anisotropy filed upon annealing of the film in a magnetic field. With an increase in the time of annealing of the low-coercivity film, the initial anisotropy decreases to zero and then grows in the applied field direction. Annealing of the high-coercivity film in a magnetic field perpendicular to the initial easy magnetization axis at $\mathrm{T}=300^{\circ} \mathrm{C}$ did no noticeably change the initial anisotropy value.

\section{Discussion}

The relaxation character of the variation in the anisotropy field shows that the induced anisotropy in the films fabricated at the high $\mathrm{pH}$ values originates from the diffusion processes of directional ordering. These processes are most frequently described within the theory of redistribution of pairs of magnetic ions along the preferred direction formed by an applied field. In our case, the diffusion processes are related to the redistribution of axes connecting the dipolar configurations of $\mathrm{Co}$ ions upon variation in the magnetization orientation under the action of a magnetic field.

The induced anisotropy relaxation time is determined by the Arrhenius ratio

$$
\tau=\tau_{0} \cdot \exp \left(\mathrm{E}_{\mathrm{A}} / \mathrm{k} T\right)
$$

where $\mathrm{E}_{\mathrm{A}}$ is the activation energy, $\mathrm{k}$ is the Boltzmann constant, and $\tau_{0}$ is the inverse probability of hoppings at high temperatures.

At room temperature, the Co-P film is characterized by $\tau \sim 200$ days [4], while at $\mathrm{T}=100^{\circ} \mathrm{C}$, by $\tau \sim 3 \mathrm{~h}$. Then, we have $\mathrm{E}_{\mathrm{A}} \sim 1 \mathrm{eV}$ and $\tau_{0} \sim 10^{-9}$.

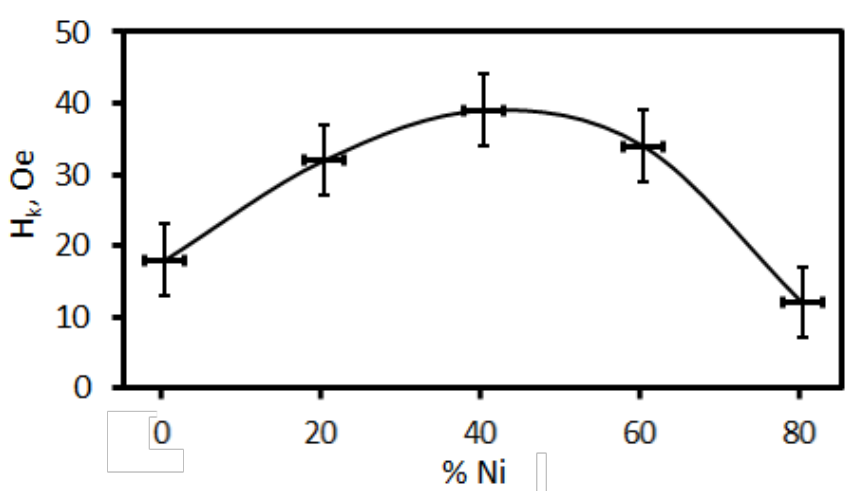

Fig. 3. Dependence of the $\mathrm{K}_{\mathrm{U}}$ value on the Ni content.

The pair mechanism is confirmed by the change in the $\mathrm{H}_{\mathrm{K}}$ value upon embedding of $\mathrm{Ni}$ ions in the $\mathrm{Co}$ lattice. As the Ni content increases to $\sim 50 \%$, the $\mathrm{H}_{\mathrm{K}}$ value grows and then, with a further increase in the $\mathrm{Ni}$ content, drops (Fig. 3). The observed changes in the anisotropy field in the pair ordering model are explained by the fact that the contribution of pairs of different ions to the anisotropy is larger than the contribution of pairs of identical ions $[5,6]$.

The non-relaxation character and high $\mathrm{K}_{U}$ values in the films obtained at the low $\mathrm{pH}$ values (up to $18 \%$ of $\mathrm{K}_{\mathrm{U}}$ for pure cobalt, which amounts to $3.3 \times 10^{6} \mathrm{erg} / \mathrm{cm}^{3}$ [7]) are indicative of the fact that the induced anisotropy is stimulated, in this case by another mechanism. According to [8], it can be (i) the growth anisotropy, (ii) elastic strains, or (iii) structural changes in the cobalt lattice upon variation in the solution acidity.
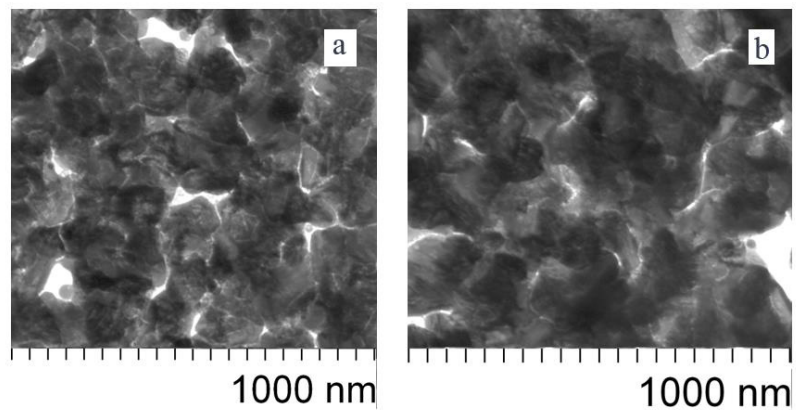

Fig. 4. TEM image of the high-coercivity films deposited in (a) zero and (b) nonzero magnetic field.

The uniaxial magnetic anisotropy caused by the directional film growth in a magnetic field was observed in the $\mathrm{Co}-\mathrm{P}-\mathrm{Ni}$ films prepared using the electrolytic technique [9]. This anisotropy is caused by the Lorentz forces that influence moving ions from the side of magnetic field and ensure the directional growth of cobalt crystallites.

Comparing electron microscopic photos of the CoP films' surfaces (Fig 4a, 4b), manufactured by the method of chemical deposition with or without magnetic field, let us conclude that magnetic field does not influence on shape and size of the crystallites. In both cases crystallites are of irregular shape caused by the island character of film's growing [3]. 
The established differences between the films grown by the chemical and electrolytic techniques are related to the fundamental difference between the mechanisms of film deposition onto a substrate. During chemical deposition, the ionic current only flows in the solutionsubstrate contact region and its value is very low.

The stresses induced during the film growth in a magnetic field can be caused by the occurrence of large crystallites and film structure compacting due to disappearance of spacers (Fig. 4). In this case, the stressinduced anisotropy constant is $\mathrm{K}_{\mathrm{U}}=3 \lambda \sigma$. The magnetostriction constant $\lambda$ of hcp Co is $\sim 10^{-5}$ [10]. Then, at $\mathrm{K}_{\mathrm{U}}=6 \times 10^{5} \mathrm{erg} / \mathrm{cm}^{3}$ the stresses $\sigma$ should attain $\sim 10^{10} \mathrm{dyn} / \mathrm{cm}^{2}$, which is beyond the film strength limits.

As was mentioned above, the $\mathrm{K}_{U}$ value significantly changes in the region of the transition of the film structure from submicrocrystalline to nanocrystalline. The structural transformations are the probable reason of the sharp drop of the induced anisotropy in the Co-Pfilms with increasing $\mathrm{pH}$ value. To fully understand this mechanism, a more detailed structural examination should be performed.

\section{Conclusions}

Based on the data obtained, we can make the following conclusions. The observed changes in the induced anisotropy in the chemically deposited Co-P films are related to the structural variations determined by the $\mathrm{pH}$ values of the solutions used. The sharp difference between the $\mathrm{K}_{\mathrm{U}}$ values and anisotropy relaxation times is indicative of the existence of different physical mechanisms responsible for the occurrence of the induced anisotropy of the Co-P films at low and high $\mathrm{pH}$ values.

\section{References}

1. A. Brenner and G. Riddell, J. Res. Nat. 37, 31 (1947)

2. A. V. Chzhan, T. N. Patrusheva, S. A. Podorozhnyak, V. A. Seredkin, and G. N. Bondarenko, Bull Russ. Acad. Sci. Phys. 80, $11(2016)$

3. A. V. Chzhan, S. A. S.A.Podorozhnyak, G. N. Bondarenko, M. N. Volochaev, and G. S. Patrin, Phys. Sol. St. 59, 7 (2017)

4. A. V. Chzhan, G. S. Patrin, S. Ya. Kiparisov, and V. A. Seredkin, J. Siberian University. Mathematics and Physics 3, 1 (2010)

5. S. Taniguchi, Sci. Rep. Res. Inst. Tohoku Univ. 7A, 269 (1955)

6. L. Neel, J. Appl. Phys. 30, 4 (1959)

7. S. Chikazumi, Physics of Ferromagnetism, M. (1987), 497 p.
8. A. G. Lesnik, Induced Magnetic Anisotropy, Kiev, Naukova dumka (1976), 163 p.

9. V. Georgescu and M. Daub, Surf. Sci. 600, 4195 (2006)

10. A. W. Simpson and D. R. Brambley, Phys. Stat. Sol. (b) 43, 291 (1971) 